\title{
Computed tomographic scanning of the lung in patients with allergic bronchopulmonary aspergillosis and in asthmatic patients with a positive skin test to Aspergillus fumigatus
}

\author{
R M Angus, M-L Davies, M D Cowan, C McSharry, N C Thomson
}

\begin{abstract}
Background - Allergic bronchopulmonary aspergillosis is a disease of asthmatic patients which may follow a protracted course and result in chronic lung damage such as central bronchiectasis. In asthma uncomplicated by allergic bronchopulmonary aspergillosis, in particular in asthmatic patients with immediate hypersensitivity type skin reactions to Aspergillus fumigatus, the incidence of bronchiectasis is uncertain.
\end{abstract}

Methods - Computed tomographic (CT) scans were performed in 17 asthmatic patients of mean (SE) age $60 \cdot 1(2 \cdot 5)$ years, $\mathrm{FEV}_{1} 49 \cdot 4(5 \cdot 8) \%$ predicted with allergic bronchopulmonary aspergillosis (all with current or previous positive precipitins to $A$ fumigatus) and in 11 asthmatic patients of mean (SE) age $49 \cdot 5(5 \cdot 8)$ years, FEV $_{1} 75.5(6.5) \%$ predicted, skin test positive for $A$ fumigatus, but without the clinical or serological features of allergic bronchopulmonary aspergillosis (nonallergic bronchopulmonary aspergillosis group).

Results - Bronchial dilatation was more common in the group with allergic bronchopulmonary aspergillosis, affecting 14 patients compared with two in the nonallergic bronchopulmonary aspergillosis group. Evidence of bronchiectasis was found in 43 of a possible 102 lobes of patients with allergic bronchopulmonary aspergillosis, compared with three of a possible 66 in the non-allergic bronchopulmonary aspergillosis group. Bronchial wall thickening was common to both, affecting 16 and nine patients respectively. Pleural thickening on CT scanning was common in the group with allergic bronchopulmonary aspergillosis, being noted in 14 patients compared with only three in the non-allergic bronchopulmonary aspergillosis group.

Conclusions - Bronchiectasis is common in allergic bronchopulmonary aspergillosis but occurs only occasionally in asthmatic patients with a positive skin test to $A$ fumigatus but without other features of the disease.

(Thorax 1994;49:586-589)
Allergic bronchopulmonary aspergillosis is a disease of asthmatic patients characterised by a potent humoral immune response to Aspergillus fumigatus. It may follow a protracted course and may progress to result in chronic lung damage such as central bronchiectasis or pulmonary fibrosis. ${ }^{1-3}$

Until recently it has been generally accepted that central bronchiectasis was virtually pathognomonic for the diagnosis of allergic bronchopulmonary aspergillosis and not a feature of uncomplicated asthma. This view has been challenged by Neeld $e t ~ a l^{4}$ who found evidence of bronchiectasis in $41 \%$ of lobes in a group of patients with allergic bronchopulmonary aspergillosis, and in $15 \%$ of lobes in asthmatic patients with a positive skin test for $A$ fumigatus but without other features of allergic bronchopulmonary aspergillosis. Paganin et $a l^{5}$ also found some degree of bronchiectasis on computed tomographic (CT) scanning in $56 \%$ of asthma patients with no evidence of $A$ spergillus allergy. Clearly, if this were the case it would have implications for the treatment and evaluation of therapies in chronic asthma.

Narrow section CT scanning is the investigation of choice in the diagnosis of bronchiectasis. It is superior to plain chest radiography in detecting bronchiectasis ${ }^{46}$ and, in comparison with bronchography which is considered unsafe in asthma, ${ }^{7}$ has a sensitivity in some series approaching $95 \%$ and a specificity of around $90 \%{ }^{8}$ We have applied CT scanning to a group of patients with allergic bronchopulmonary aspergillosis and to a group of asthmatic patients with a positive skin test to $A$ fumigatus but without other features of allergic bronchopulmonary aspergillosis. Our aim was to determine the extent of bronchiectatic changes in allergic bronchopulmonary aspergillosis and to establish whether or not a similar spectrum of destructive changes is common in the other asthmatic patients.

\section{Methods}

PATIENTS

Patients were identified from case records and were seen prospectively. All patients were asthmatic and demonstrated a positive skin prick test to $A$ fumigatus. Allergic bronchopulmonary aspergillosis was diagnosed if, in addition, the patients had positive precipitins to $A$ 
fumigatus and had a history of a flare in their symptoms associated with a positive sputum culture for $A$ fumigatus. Radiological features were not used in patient selection. Seventeen patients with allergic bronchopulmonary aspergillosis of mean (SE) age $60 \cdot 1(2 \cdot 5)$ years, $\mathrm{FEV}_{1} 1.3(0 \cdot 14)$ litres and $49.4(5.8) \%$ predicted, and 11 asthmatic patients with a positive skin test to allergic bronchopulmonary aspergillosis but with no other features of the disease of mean age $49.5(4.5)$ years, $\mathrm{FEV}_{1} 2 \cdot 1$ $(0.3)$ litres and $75.5(6.5) \%$ predicted were studied. Patient characteristics are detailed in table 1 .

\section{CT SCANNING}

A Philips Tomoscan 310 with a 9.5 second scan time was used. Thin section studies were performed using a $3 \mathrm{~mm}$ slice thickness scanning at $9 \mathrm{~mm}$ increments and the images were reconstructed using a high spatial frequency algorithm. The CT images were analysed separately by two senior radiologists in a blinded fashion, differences being resolved by consensus.

Bronchiectasis was deemed to be present if the bronchial diameter was greater than that of the accompanying artery ${ }^{910}$ and was defined as being central when confined to the medial two thirds of the lung. The degree of bronchiectasis was also assessed, being allocated to one of three categories: mild - where the bronchial diameter was only slightly larger than the accompanying artery; marked - where the dilatation was obvious; and cystic - where the bronchi showed cystic dilatation.

Bronchial wall thickening was evaluated by comparison with areas of normal lung in any individual. ${ }^{910}$ Other features including pleural thickening, parenchymal shadowing, volume loss, and emphysematous changes were also noted.

Each lobe in every patient was analysed separately.

\section{CHEST RADIOGRAPHS}

Posteroanterior chest radiographs were also performed at the time of scanning. These were

Table 1 Patient characteristics expressed as mean ( $S E)$ values

\begin{tabular}{|c|c|c|}
\hline & $\begin{array}{l}A B P A \\
(n=17)\end{array}$ & $\begin{array}{l}\text { Non-ABPA } \\
(n=11)\end{array}$ \\
\hline $\begin{array}{l}\text { M:F } \\
\text { Age (years) } \\
\text { Total IgE (mg/ml) } \\
\text { Asthma (years) } \\
\text { Diagnosis of ABPA (years) } \\
\text { Inhaled corticosteroids } \\
\text { Oral prednisolone } \\
\text { Current/ex-smoker } \\
\text { FEV }_{1} \text { (litres) } \\
\text { FEV (\% predicted) } \\
\text { RAST IgE to } \text { A fumigatus } \\
\text { (IU/ml) } \\
\text { Specific IgG to } \text { A fumigatus } \\
\text { (U)/ml) } \\
\text { ECP }(\mu \mathrm{g} / \mathrm{l})\end{array}$ & $\begin{array}{l}12: 5 \\
60 \cdot 1(2 \cdot 5) \\
676(169) \\
27 \cdot 6(5 \cdot 3) \\
7 \cdot 5(1 \cdot 5) \\
16 \\
6 \\
11 \\
1 \cdot 3(0 \cdot 14) \\
49 \cdot 4(5 \cdot 8) \\
4 \cdot 6(4 \cdot 2) \\
41.9(16 \cdot 7) \\
17(2 \cdot 5)\end{array}$ & $\begin{array}{l}5: 6 \\
49 \cdot 5(4 \cdot 5) \\
387(429) \\
23 \cdot 2(5 \cdot 4) \\
-\quad \\
7^{*} \\
1 \\
4 \\
2 \cdot 1(0 \cdot 3)^{*} \\
75 \cdot 5(6 \cdot 5)^{*} \\
1 \cdot 32(1 \cdot 0)\end{array}$ \\
\hline
\end{tabular}

reported separately from the CT scans, again in a blinded fashion and independently by the two radiologists. Bronchiectasis was diagnosed using the signs described by McCarthy et al, ${ }^{11}$ the following changes being accepted as bronchiectasis: parallel line shadows, band-like (tooth paste) shadows, gloved finger shadows, and ring shadows.

\section{DATA ANALYSIS}

Differences between groups were compared by the $\chi^{2}$ test and continuous variables by the Mann-Whitney $U$ test. The relation of IgE, IgG, and $F E V_{1}$ with the grades of bronchiectasis was compared by analysis of variance. A p value of $<0.05$ was taken as being significant. Analysis was performed on an Amstrad personal computer using the Minitab software package.

\section{Results}

Fourteen of the 17 patients with allergic bronchopulmonary aspergillosis had evidence of bronchiectasis on CT scanning compared with two of the 11 in the group without allergic bronchopulmonary aspergillosis $(p<0.05)$. This difference was more significant when the number of lobes affected was considered. Evidence of bronchiectasis was found in 43 $(42 \%)$ of a possible 102 lobes in the group with allergic bronchopulmonary aspergillosis compared with three $(5 \%)$ of a possible 66 in the non-allergic bronchopulmonary aspergillosis group. When grades of bronchiectasis were compared they were found by inspection to be related to the specific IgE and IgG $(p<0.05)$, but not to the $\mathrm{FEV}_{1}$. Bronchial dilatation was found to be central in most patients (figure, table 2); one patient had both central and peripheral changes. Of the 16 patients noted to have bronchiectasis on CT scanning, only 12 were detected on the chest radiograph (table 2).

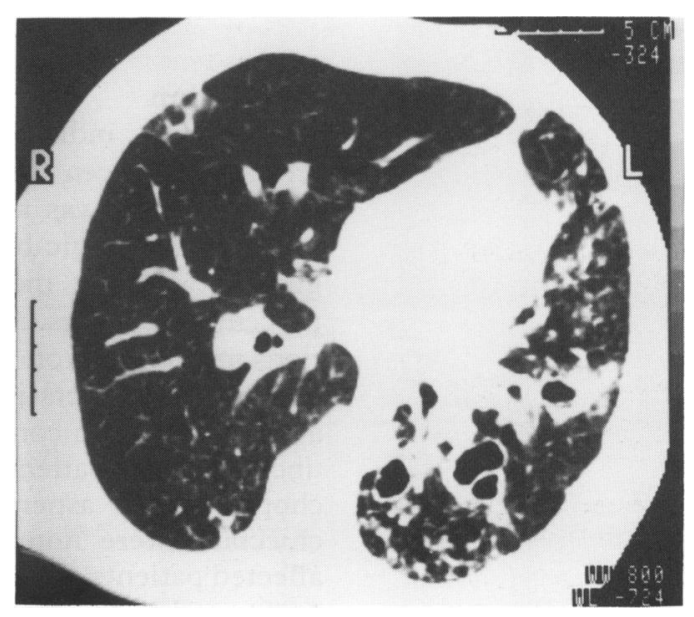

Central (proximal) bronchiectasis in a patient with allergic bronchopulmonary aspergillosis. $A$ high resolution $C T$ scan of the chest showing bronchiectatic changes in the left lower lobe. The bronchi are larger than the adjacent artery and their walls are thickened compared with the other side. 
Table 2 Distribution and severity of computed tomographic scanning changes of bronchiectasis in 17 patients with $A B P A$ and 11 patients with a positive skin test for A fumigatus but without the clinical or serological features of $A B P A$ (non- $A B P A$ group)

\begin{tabular}{lll}
\hline & $\begin{array}{l}A B P A \\
(n=17)\end{array}$ & $\begin{array}{l}\text { Non- } A B P A \\
(n=11)\end{array}$ \\
\hline $\begin{array}{l}\text { Bronchiectasis } \\
\text { No. of patients } \\
\begin{array}{l}\text { No. of cases diagnosed by } \\
\text { chest radiograph }\end{array}\end{array}$ & 14 & $2^{*}$ \\
$\quad$ Lobes affected (total) & $43(102)$ & $2^{*}$ \\
$\begin{array}{l}\text { Distribution } \\
\quad \text { Central (proximal) }\end{array}$ & 39 & 3 \\
$\quad \begin{array}{l}\text { Peripheral } \\
\text { Degree of bronchiectasis }\end{array}$ & 5 & 0 \\
$\quad$ Mild & 27 & 1 \\
$\quad$ Marked & 10 & 2 \\
Cystic & 6 & 0 \\
\hline
\end{tabular}

$\mathrm{ABPA}=$ allergic bronchopulmonary aspergillosis. ${ }^{*} \mathrm{p}<0.05$.

Table 3. Abnormalities other than bronchiectasis seen on computed tomographic scanning in 17 patients with $A B P A$ and 11 patients with a positive skin test for $A$ fumigatus but without the clinical or serological features of $A B P A$ (non- $A B P A$ group)

\begin{tabular}{lll}
\hline & $\begin{array}{l}A B P A \\
(n=17)\end{array}$ & $\begin{array}{l}\text { Non- } A B P A \\
(n=11)\end{array}$ \\
\hline Bronchial wall thickening & 16 & 9 \\
Pleural thickening & 14 & $3^{*}$ \\
Parenchymal shadowing & 14 & $5^{*}$ \\
Collapse/volume loss & 9 & 2 \\
Emphysema & 9 & $1^{*}$
\end{tabular}

ABPA = allergic bronchopulmonary aspergillosis. $* \mathrm{p}<0.05$.

Bronchial wall thickening was common in both groups of patients affecting 16 patients in the group with allergic bronchopulmonary aspergillosis and nine patients in the group without allergic bronchopulmonary aspergillosis (table 3). The presence of pleural thickening on CT scanning was common in the group with allergic bronchopulmonary aspergillosis (14 patients) compared with only three in the other group (table 3). Parenchymal shadowing and emphysematous changes were more common in the group with allergic bronchopulmonary aspergillosis than in those without the condition (table 3 ).

\section{Discussion}

Our findings indicate several important differences between the two groups studied. Bronchiectasis was a common feature of the group with allergic bronchopulmonary aspergillosis, affecting most patients and a large percentage of lobes. As with previous descriptions in allergic bronchopulmonary aspergillosis, the bronchiectasis noted was mainly situated centrally. In contrast, bronchiectasis was uncommon in patients without allergic bronchopulmonary aspergillosis. Signs of bronchiectasis were noted in only 12 of the 16 affected patients on the plain chest radiographs performed at the same time as the CT scans, re-emphasising the greater sensitivity of CT scanning. ${ }^{69}$

In the patients with allergic bronchopulmonary aspergillosis bronchial dilatation was found in similar numbers of patients and lobes as was reported in the studies by Neeld et al and Currie et al. ${ }^{46}$ In contrast, bronchiectasis was found in only $5 \%$ of lobes of patients without allergic bronchopulmonary aspergillosis, whereas Neeld reported this in $15 \%$ of lobes in a similarly defined group. ${ }^{4}$ There may be some differences between their patients and those of the present study as the three patients with bronchiectasis in their group of eight non-allergic bronchopulmonary aspergillosis patients were taking oral corticosteroids, suggesting that they had more severe asthma; neither of our two non-allergic bronchopulmonary aspergillosis patients with bronchiectasis were taking oral corticosteroids. Our findings are in even more marked contrast to the findings of Paganin et $a l^{5}$ who recently reported that there was evidence of bronchiectasis on CT scanning in $56 \%$ of asthmatic patients across a varying severity of disease. The patients in their study were different in that none were smokers and none had evidence of hypersensitivity to A fumigatus. Similarly, some of their patients were scanned when acutely unwell which may exaggerate the incidence of bronchiectasis if acute transient dilatation occurred which resolved with treatment. Nevertheless, our findings would not corroborate their frequent finding of bronchiectasis in asthmatic patients. We also think it is unlikely that technical differences would account for this disparity although different section thicknesses and increments were used; Neeld used $1 \mathrm{~mm}$ sections at $15 \mathrm{~mm}$ intervals and Paganin used $1.5 \mathrm{~mm}$ sections at 10 or $20 \mathrm{~mm}$ intervals, compared with $3 \mathrm{~mm}$ sections at $9 \mathrm{~mm}$ intervals used in our study. Likewise, although there is a degree of subjectivity in the interpretation of scans, it seems unlikely that this would account for the differences reported as the same criteria for bronchial dilatation were used.

Pleural thickening was common in the patients with allergic bronchopulmonary aspergillosis but infrequent in those without the disease. Pleuritic chest pain has been described in allergic bronchopulmonary aspergillosis $^{11}$ although, to our knowledge, pleural thickening has not been reported. The finding of pleural thickening presumably reflects changes following episodic pleural inflammation; it should be noted, however, that this was relatively minor and unlikely to be of clinical significance. Bronchial wall thickening was common to both groups as has previously been described $^{45}$ and highlights the inflammatory nature of asthma. Emphysematous changes were more common in the patients with allergic bronchopulmonary aspergillosis which is in keeping with the more severe degree of airflow obstruction noted in this group.

We conclude from our findings that bronchiectasis is common in allergic bronchopulmonary aspergillosis but occurs only occasionally in asthmatic patients with a positive skin test to $A$ fumigatus. We would contend, therefore, that the presence of bronchiectasis should continue to be regarded as a diagnostic feature suggestive of allergic bronchopulmonary aspergillosis and should not be accepted 
simply as a common feature of asthma. In the evaluation of patients CT scanning is therefore useful if allergic bronchopulmonary aspergillosis is considered as a possible diagnosis.

This study was supported by a grant from the Chest, Heart and Stroke Association (Scotland).

1 Malo JL, Hawkins R, Pepys J. Studies in chronic allergic bronchopulmonary aspergillosis. Three immunological findings. Thorax 1977:32:254-61.

2 Greenburger PA, Patterson R, Chong AC, Arkin JA, Walsh $\mathrm{T}$, Graves $\mathrm{T}$, et al. Late sequalae of allergic bronchopulmonary aspergillosis. F Allergy Clin Immunol 1980; 66:327-30.

3 Greenburger PA, Patterson R. Allergic bronchopulmonary aspergillosis and the evaluation of the patient with asthma. F Allergy Clin Immunol 1988;81:646-50.

4 Neeld DA, Goodman LR, Gurney JW, Greenberger PA, Fink JN. Computed tomography in the evaluation of allergic bronchopulmonary aspergillosis. Am Rev Respir Dis $1990 ; 142: 1200-5$.
5 Paganin F, Trussard V, Seneterre E, Chanez P, Giron J, Godard $P$, et al. Chest radiography and high resolution computed tomography of the lungs in asthma. Am Rev Respir Dis 1992;146:1084-7.

6 Currie DC, Goldman JM, Cole PJ, Strickland B. Comparison of narrow section computed tomography and plain chest radiography in chronic allergic bronchopulmonary aspergillosis. Clin Radiol 1987;38:593-6.

7 Fraser RG, Pare JAP. Methods of roentogenologic and pathologic investigation. In: Diagnosis of diseases of the pathologic investigation. In: Diagnosis of
chest. Philadelphia: Saunders, 1977:211-8.

8 Grenier P, Maurice F, Musset D, Menu Y, Nahum H. Bronchiectasis: assessment by thin-section CT. Radiology 1986;161:95-9.

9 Naidich DP, McCauley DI, Khouri NF, Stitik FP, Siegelman SS. Computed tomography of bronchiectasis. $\mathcal{F}$ Comput Assist Tomogr 1982;6:437-44.

10 Munro NC, Cooke JC, Currie DC, Strickland B, Cole PJ. Comparison of thin section computed tomography with bronchography for identifying bronchiectatic segments in patients with chronic sputum production. Thorax 1990;45:135-9.

11 McCarthy DS, Simon G, Hargreave FE. The radiological appearances in allergic bronchopulmonary aspergillosis. Clin Radiol 1970;21:366-75. 